\title{
Visualization Analysis of Numerical Solution With 32x32 and 64x64 Mesh Grid Lid-Driven Square Cavity Flow
}

\author{
Santhana Krishnan Narayanan ${ }^{1}$, Antony Alphonnse Ligori ${ }^{2}$ and Jagan Raj ${ }^{3}$ \\ ${ }^{1}$ PG and Research Department of Computer Science, Dr. Ambedkar Government Arts College, Chennai, Tamilnadu, India \\ ${ }^{2}$ Department of Mathematics, Gedu College of Business Studies, Royal University of Bhutan, Bhutan \\ ${ }^{3}$ Department of Chemistry, Velammal Institute of Technology, Panjetty, Tamil Nadu, India
}

\begin{abstract}
In this paper, the flow problem of constant velocity of a square cavity whose lid is solved and obtained a numerical solution on 2 grid levels, having $32 \times 32$ and $64 \times 64$ cells. Reynolds number of $1 \times 10^{2}, 1 \times 10^{3}$ was selected for laminar flow and $8 \times 10^{3}$ was selected for turbulent flow. The problem is identified in NavierStokes equations. The finite volume discretization is based on the numerical model. The simulated results are in valid agreement with those that are available in the given report. The numerical solution of these works are accurately obtained for this problem.
\end{abstract}

Keywords:- Navier-Stokes Equation, Finite volume method, CFD, Discretization, Internal flow.

\section{INTRODUCTION}

The recent researches have witnessed a great deal of progress in Lid-driven square cavity flow problems. Developments in computer technology hardware with $10^{\text {th }}$ Generation Intel ${ }^{@}$ core $^{*} \mathrm{i} 7$ processor workstation computer system as well as in the analysis and numerical solution of highly complex flow problems advanced numerical algorithms have enabled the attempts to be made towards various lists such as Lid-driven square cavity. Harlow et al. [1] developed a new method for the boundary of which is partially confined and partially free numerical investigation of the time dependent flow of an incompressible fluid. Ghia et al.[2] investigated the vorticity-stream function formulation of the two-dimensional Navier-Stokes incompressible equations and used the efficacy of the Coupled Highly Implicit Multi Grid (CSI-MG) approach to evaluate high-refine-mesh flow solutions. Carbs et al.[3] solved the flow problem inside a square cavity, whose lid has constant pace. Zhang[4] found that multi-grid techniques are used to model the two-dimensional squaredriven cavity flow with small to large Reynolds numbers in fourth-order compact finite difference schemes. Erturk et al.[5] examined the 2-D steady incompressible driven cavity flow numerical calculations. The present study represents an effort to employ the multigrid method in the solution of the Navier-Stokes equation for a Lid-driven square cavity flow problem with a goal of obtaining solutions for Reynolds numbers and mesh refinements as high as possible.

\section{> Mathematical Model}

The considered physical system has a steady state two dimensional (2-D) length $\mathrm{L}$ of lid-driven square cavity is represented in figure 1 . The flow inside a square cavity of which the top wall (lid) is moving with a uniform velocity. Here, $\mathrm{u}$ and $\mathrm{v}$ are the parameters of the velocity vectors $\mathrm{x}$ and $y$ directions, $\rho$ and $\mu$ are the fluid density and their constant viscosity.

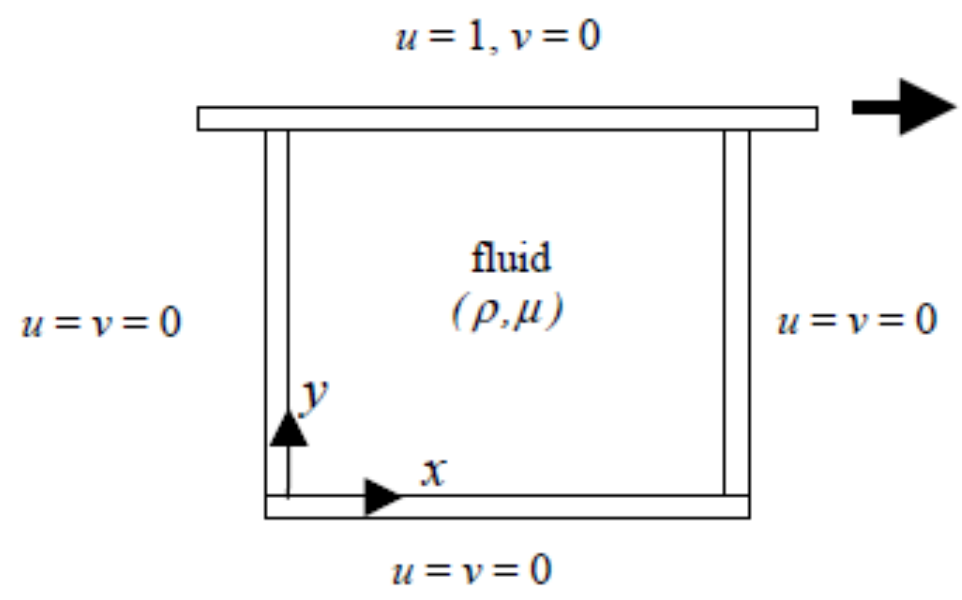

Fig 1:- Physical system of Lid-driven Square Cavity 
The incompressible two dimensional Navier-Stokes equation with the lid velocity $\mathrm{U}_{\text {lid }}$, the mass and momentum equations can be written in dimensionality form as

$$
\frac{\partial u^{\prime}}{\partial x^{\prime}}+\frac{\partial v^{\prime}}{\partial y^{\prime}}=0
$$

$\rho \frac{\partial u^{\prime}}{\partial t^{\prime}}+\rho u^{\prime} \frac{\partial u^{\prime}}{\partial x^{\prime}}+\rho v^{\prime} \frac{\partial u^{\prime}}{\partial y^{\prime}}=-\frac{\partial p^{\prime}}{\partial x^{\prime}}+\left(\frac{\mu \partial^{2} u^{\prime}}{\partial x^{\prime}}+\frac{\mu \partial^{2} u^{\prime}}{\partial y^{\prime 2}}\right)$

$\rho \frac{\partial v^{\prime}}{\partial t^{\prime}}+\rho u^{\prime} \frac{\partial v^{\prime}}{\partial x^{\prime}}+\rho v^{\prime} \frac{\partial v^{\prime}}{\partial y^{\prime}}=-\frac{\partial p^{\prime}}{\partial y^{\prime}}+\left(\frac{\mu \partial^{2} v^{\prime}}{\partial x^{\prime}}+\frac{\mu \partial^{2} v^{\prime}}{\partial y^{\prime 2}}\right)$

After non-dimensionalising, the mass and momentum equations can be written as

$$
\begin{gathered}
\frac{\partial u}{\partial x}+\frac{\partial v}{\partial y}=0 \\
\frac{\partial u}{\partial t}+u \frac{\partial u}{\partial x}+v \frac{\partial u}{\partial y}=-\frac{\partial p}{\partial x}+\frac{1}{R e}\left(\frac{\partial^{2} u}{\partial x^{2}}+\frac{\partial^{2} u}{\partial y^{2}}\right) \\
\frac{\partial v}{\partial t}+u \frac{\partial v}{\partial x}+v \frac{\partial v}{\partial y}=-\frac{\partial p}{\partial y}+\frac{1}{R e}\left(\frac{\partial^{2} v}{\partial x^{2}}+\frac{\partial^{2} v}{\partial y^{2}}\right)
\end{gathered}
$$

Here $=\frac{u^{\prime}}{U_{\text {lid }}},=\frac{v^{\prime}}{U_{\text {lid }}}, x=\frac{x^{\prime}}{L_{\text {lid }}}, y=\frac{y^{\prime}}{L_{\text {lid }}}, t=t^{\prime} \frac{U_{\text {lid }}}{L_{\text {lid }}}, \rho=\frac{\rho^{\prime}}{\rho_{\text {ref }}}$ $, \mu=\frac{\mu^{\prime}}{\mu_{\text {ref }}} \quad, p=\frac{p^{\prime}-p_{\text {ref }}}{\rho U_{\text {lid }}^{2}} \quad$ with Re being the Reynolds number, $R e=\frac{\rho^{\prime} U_{\text {lid }} L_{\text {lid }}}{\mu^{\prime}}$ and $p$ is the pressure. No slip boundary condition has been applied for $u$ and $v$ directions at bottom and side walls as

$$
u=0 \text { and } v=0
$$

and in the top wall

$$
u=U_{\text {lid }}=1 \text { and } v=0
$$

Second Ordered Central (SOC) with finite volume method is fixed to discretize the governing functions and finite volume operator on the $x$-momentum and $y$ momentum equations are utilized to obtain the numerical solutions. Pre and Post processing is done using Matlab software.

\section{RESULTS AND DISCUSSIONS}

\section{Mesh Grid $32 \times 32$}

The lid-driven square cavity flow relates to the twodimensional flow evolution as the wall velocity increases. In the absence of instability, the steady, two-dimensional flow should develop into a vortex with a viscid center of uniform vorticity surrounded by viscous boundary layers that connect the vortex center to the boundary conditions for large Reynolds numbers.However, At large Reynolds numbers, the two-dimensional steady flow is not stable and smaller scale vortices are shed from the downstream end of the moving wall into the cavity when the Reynolds number rises above a critical value.

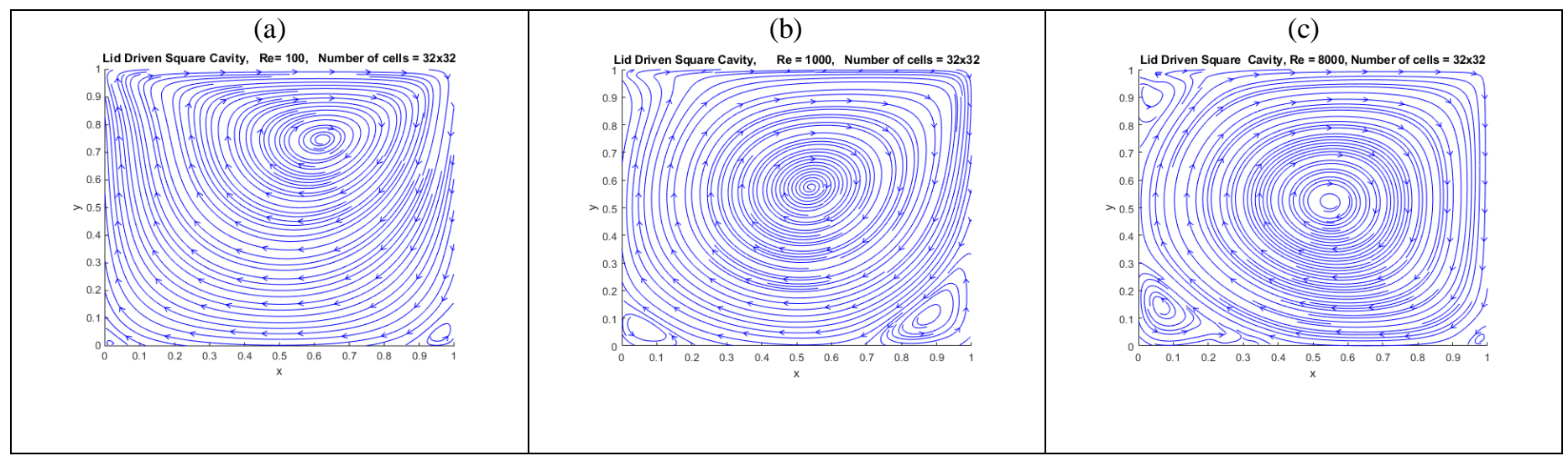

Fig 2:- Streamlines of the stokes stream function in a lid-driven square cavity with $32 \times 32$ grid $(a) \operatorname{Re}=100$ (b) $R e=1000$ and (c) $\operatorname{Re}=8000$

Figure 2 displays standard streamline patterns of the two-dimensional global re-circulating vortex powered by the moving wall with a $32 \times 32$ grid for $\mathrm{Re}=100,1000$ and 8000. In the case of $\mathrm{Re}=100$ shown in Figure 2a, the streamlines are almost symmetrical due to the symmetries of the governing equation in the flow limit of stocks $\operatorname{Re} \rightarrow 0$. In Figure $3 a$, the contour visualization of the $\mathrm{x}$-momentum stokes stream function along the $\mathrm{u}$-direction is shown. Figure 4a also demonstrates the contour visualization of the $y$-momentum stokes stream function along v-direction. Near the moving lid, where the greatest velocities emerge, the streamlines are slightly crowded, and two distinct eddies in the lower corners are signaled by the two separating threads. 


(a)

Fig 3:- Contour Visualization of the stokes stream function in $\mathrm{x}$-momentum along $\mathrm{u}$ direction in a lid-driven square cavity with $32 \times 32$ grid (a) $R e=100$ (b) $\operatorname{Re}=1000$ and (c) $\operatorname{Re}=8000$

For $\mathrm{Re}=1000$ shown in Figure 2b, the main vortex, whose center for high Reynolds number forms circular streamlines, drives another weaker separated and counter-rotating vortex, and so forth. Figure $3 \mathrm{~b}$ displays the contour visualization of the stokes stream function in $\mathrm{x}$-momentum along the u-direction. In addition, the contour visualization of the y-momentum stokes stream function along v-direction is shown in figure 4. While the two-dimensional flow is steady for small and moderate Reynolds numbers, as inertia impacts become strong, it undergoes a Hopf bifurcation and becomes time-dependent for higher Reynolds numbers.

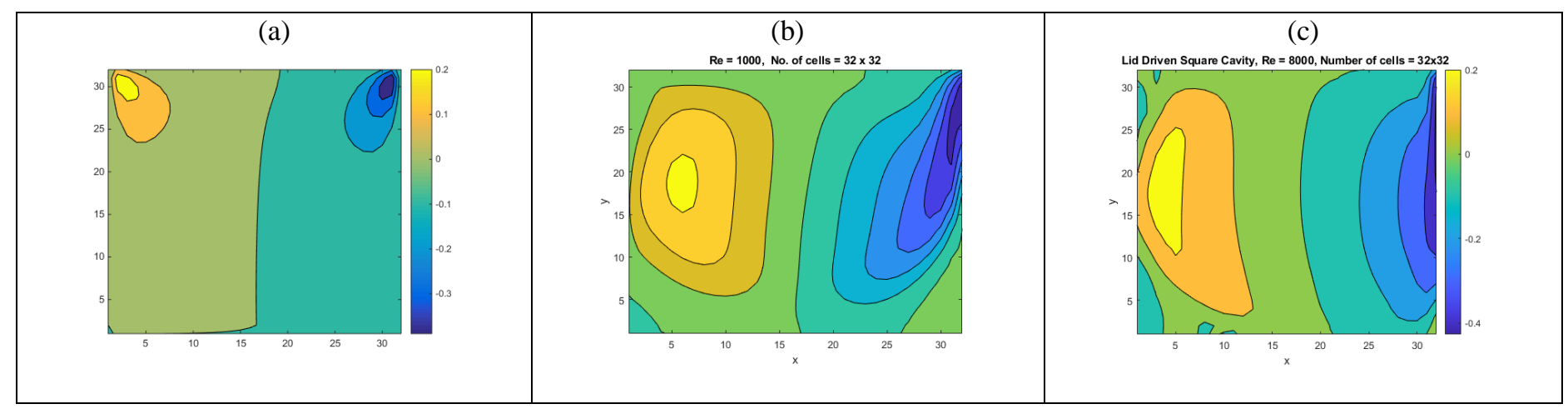

Fig 4:- Contour Visualization of the stokes stream function in function in y-momentum along v-direction in a lid-driven square cavity with $32 \times 32$ grid (a) $R e=100$ (b) $R e=1000$ and (c) $\operatorname{Re}=8000$

For the broad $\mathrm{Re}=8000$ shown in figure $2 \mathrm{c}$, inertia terms in governing equation kill the reflective symmetry relative to $\mathrm{x}=0$ of the flow. In Figure $3 \mathrm{c}$, the contour visualization of the $\mathrm{x}$-momentum stokes stream function along $u$-direction is shown. Figure $4 \mathrm{c}$ also shows contour display of the stokes stream function in y-momentum along v-direction. The separate vortices at the bottom become stronger, in the lower right corner also a second separate vortex is visible and a third separate region is formed, close to the moving lid's upstream corner. The new quaternary vortex at the bottom left corner increases with the Reynolds number, and a new tertiary vortex is observed in the flow field at the top left corner of the cavity.For this problem, detailed exact results have been visualized. The results presented here comply with [2] reported solutions for fine grids.

\section{Mesh Grid $64 \times 64$}

Typical streamline patterns of the globally recirculating two-dimensional vortex powered by the moving wall for $\mathrm{Re}=100,1000$ and 8000 with $64 \times 64$ grid are shown in figure 5. For $\mathrm{Re}=100$ shown in Figure 5a, due to the symmetries of the governing equation in the stokes flow limit $\mathrm{Re} \rightarrow 0$, the streamlines are almost symmetrical. In Figure 6a, the contour visualization of the $\mathrm{x}$-momentum stokes stream function along the u-direction is shown. Figure 7a also shows contour visualization of the stokes stream function in $y$-momentum along v-direction. Near the moving lid, where the greatest velocities emerge, the streamlines are slightly crowded, and two distinct eddies in the lower corners are signalled by the two separating threads. 


(b)

Fig 5:- streamlines of the stokes stream function in a lid-driven square cavity with $64 \times 64$ grid (a) $R e=100$ (b) $R e=1000$ and (c) $R e=8000$

For $\mathrm{Re}=1000$ shown in Figure 5b, the main vortex, whose center for high Reynolds number forms circular streamlines, drives another weaker separated and counter-rotating vortex, and so forth. In Figure $6 \mathrm{~b}$, the contour visualization of the $\mathrm{x}$ momentum stokes stream function along the u-direction is shown.Also, Contour visualization of the stokes stream function in $y$ momentum along v-direction is shown in figure $7 \mathrm{~b}$. While the two-dimensional flow for small and moderate Reynolds numbers is steady, it undergoes a Hopf bifurcation and becomes time-dependent for higher Reynolds numbers when inertia effects becomes large.

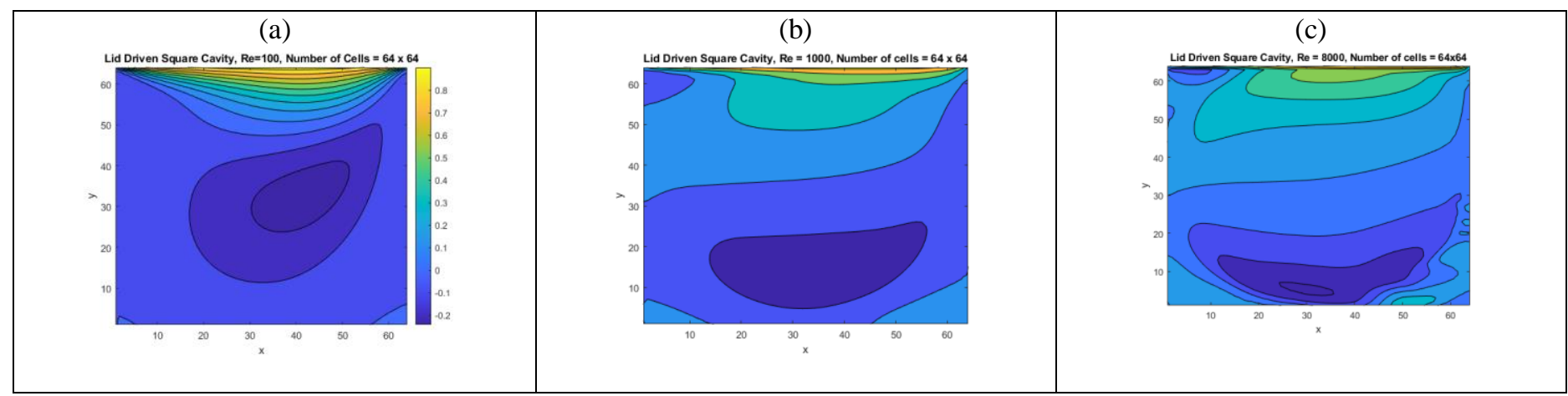

Fig 6:- Contour Visualization of the stokes stream function in $\mathrm{x}$-momentum along u-direction in a lid-driven square cavity with 64x64grid (a) $\operatorname{Re}=100$ (b) $\operatorname{Re}=1000$ and (c) $\operatorname{Re}=8000$

For the broad $\mathrm{Re}=8000$ shown in figure $5 \mathrm{c}$, inertia terms in governing equation kill the reflective symmetry relative to $\mathrm{x}=0$ of the flow. In Figure $6 \mathrm{c}$, the contour visualization of the $\mathrm{x}$-momentum stokes stream function along u-direction is shown. Figure 7c also demonstrates the contour visualization of the y-momentum stokes stream function along v-direction. The separate vortices at the bottom become stronger, in the lower right corner also a second separate vortex is visible and a third separate region is formed, close to the moving lid's upstream corner. The new quaternary vortex at the bottom left corner increases with the Reynolds number, and a new tertiary vortex is observed in the flow field at the top left corner of the cavity. For this problem, detailed results have been visualized. The results presented here comply with [2] reported solutions for fine grids.

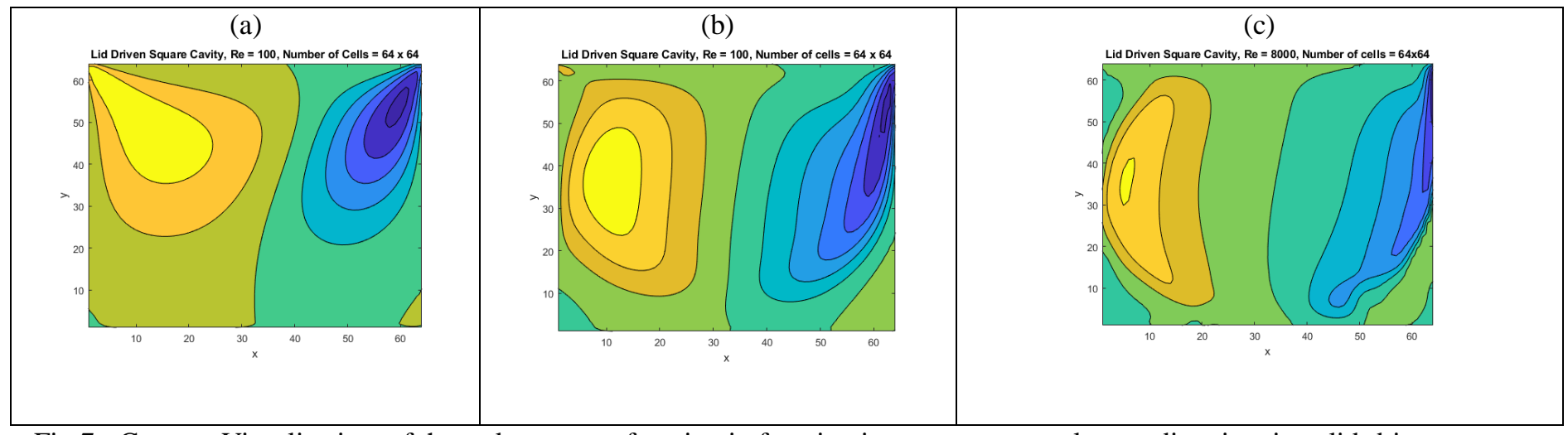

Fig 7:- Contour Visualization of the stokes stream function in function in y-momentum along v-direction in a lid-driven square cavity with 64x64grid (a) $R e=100$ (b) $R e=1000$ and (c) $R e=8000$ 


\section{CONCLUSION}

For the laminar flow with $R e=100,1000$ and turbulent flow with $R e=8000$ fine mesh solutions were obtained very efficiently. The finest mesh size used in the grid series, $32 \times 32$ and $64 \times 64$, remains a very important parameter.It was shown that the smoothing factor of the iteration schemes was affected by the Reynolds number physical problem parameters. Using this Lid-driven square cavity issue, the robustness and efficiency of the overall solution techniques has been demonstrated. For this question, comprehensive specific outcomes have been presented. The present findings comply with published fine-grid solutions.

\section{REFERENCES}

[1]. Harlow F.H and Welch J.E, "Numerical Calculation of Time-Dependent viscous incompressible flow of fluid with free surface", The Physics of Fluids, Vol. 3, pp. 2182-2189, 1965.

[2]. Ghia V, Ghia K and Shin C, "High resolutions for incompressible flow using the Navier-Stokes equations and a multigrid method', Journal of computational Physics, Vol. 48, No. 3, pp. 387-411, 1982.

[3]. Carbs H.M, Roberta S and Araki L.K, "The Lid-driven square cavity flow : Numerical solution with a $1024 \times 1024$ grid', Journal of the Brazil Soc. of Mech. Sci. \&Engg., Vol. XXXI, No. 3/191, pp. 186-198, 2009.

[4]. Zhang J, "Numerical simulation of $2 D$ square driven cavity using fourth-order compact finite difference schemes, Computers and Mathematics with Applications, Vol. 45, pp. 43-52, 2003.

[5]. Erturk E, Carke T.C and Gokcol, "Numerical solutions of steady incompressible driven cavity flow at high Reynolds number", International Journal of Numerical methods in Fluids, Vol. 48, pp. 747-774, 2005.

[6]. Koseff J.R, StrentR.L, "On end wall effects in a lid driven cavity flow", J. Fluids Eng., Vol. 106, pp. 385389, 1984. 